ARTIGO ORIGINAL ORIGINAL ARTICLE

\title{
Aplicação de Avaliação de Tecnologias em Saúde (ATS) na tomada de decisão em hospitais
}

\author{
Application of health technology assessment \\ (HTA) in the decision-making in hospitals
}

Fernando de Rezende Francisco' ${ }^{1}$ Ana Maria Malik'

DOI: $10.21115 / J B E S . v 11 . n 1 . p 10-7$

\section{RESUMO}

Objetivo: A incorporação de tecnologias em saúde por hospitais afeta o desempenho financeiro e assistencial do sistema de saúde de todo um país, e faltam informações na literatura sobre a aplicação de Avaliação de Tecnologias em Saúde (ATS) em hospitais no Brasil. Adicionalmente, há indícios de que a aplicação de ATS em hospitais pode melhorar o uso dos investimentos financeiros de todo o setor de saúde. O objetivo deste trabalho foi analisar a aplicação dos métodos de ATS na tomada de decisão em hospitais vinculados à Rebrats (Rede Brasileira de Avaliação de Tecnologias em Saúde). Métodos: Optou-se por utilizar o método da Grounded Theory, ou Teoria Fundamentada nos Dados, e os dados foram analisados com o uso do software ATLAS.ti. Foram realizadas entrevistas com gestores de 11 Núcleos de ATS (NATS), baseadas em roteiro semiestruturado preparado a partir dos 15 princípios orientadores para boas práticas de ATS em hospitais. Resultados: Os resultados indicaram que a ATS é aplicada de maneira incipiente no processo de tomada de decisão em hospitais. Conclusão: A análise do conteúdo das entrevistas permitiu, ainda, concluir que o sucesso dos NATS para a incorporação e desincorporação de tecnologias em saúde depende de uma cadeia de ações estratégicas relacionadas, e a falta de qualquer uma das etapas enfraquece, ou mesmo inviabiliza, a plena aplicação da ATS para a tomada de decisões em hospitais.

\section{Keywords:}

Health Technology Assessment (HTA), decision-making, hospital, Grounded Theory

\begin{abstract}
Objective: The incorporation of health technologies by hospitals affects the financial and healthcare performance of the health system of a whole country, and there is a lack in literature about the use of Health Technology Assessment (HTA) for decision making in Brazilian's hospitals. In addition, there is evidence that the application of HTA in hospitals can improve the use of financial investments of the whole health sector. The objective of this study was to analyze the application of HTA methods in decision making in hospitals linked to REBRATS (Brazilian Network for the Health Technologies Assessment). Methods: Grounded Theory methodology has been applied, and the interviews data were analyzed using the ATLAS.ti software. Interviews were conducted with managers of 11 hospital-based HTA units (called "NATS", Núcleo de Avaliação de Tecnologias em Saúde), based on a semi-structured script prepared from the 15 guiding principles for good practices in hospital-based HTA units. Results: The results indicated that HTA is applied in an incipient way in the decision making process in hospitals. Conclusion: The analysis of the interviews' content also allowed us to conclude that the success of the hospital-based HTA units for the incorporation and disincorporation of health technologies depends on a chain of related strategic actions, and the lack of any of the stages weakens or even impedes the full application of HTA for decision-making in hospitals.
\end{abstract}

Recebido em: 27/12/2018. Aprovado para publicação em: 04/04/2019.

1. Escola de Administração de São Paulo, Fundação Getúlio Vargas, São Paulo, SP, Brasil.

Instituição onde o trabalho foi executado: Escola de Administração de São Paulo, Fundação Getúlio Vargas, São Paulo, SP, Brasil. Informações sobre auxílios recebidos sob a forma de financiamento, equipamentos ou medicamentos: $\mathrm{O}$ autor Fernando de Rezende Francisco foi aluno bolsista da Coordenação de Aperfeiçoamento de Pessoal de Nível Superior (Capes) no curso de mestrado acadêmico, durante o qual realizou o estudo descrito neste artigo.

Congressos onde o estudo foi apresentado: Trabalho vinculado à dissertação: Francisco FR, Malik AM. Aplicação de Avaliação de Tecnologias em Saúde (ATS) e a tomada de decisão em hospitais. CALASS - Congresso da Associação Latina para a Análise dos Sistemas de Saúde. Liège, Bruxelas, Bélgica; 2017.

Premiação: Prêmio IESS (Instituto de Estudos da Saúde Suplementar), São Paulo, Brasil, 2017 pelo trabalho vinculado à dissertação: Francisco FR, Malik AM. Aplicação de Avaliação de Tecnologias em Saúde (ATS) e a tomada de decisão em hospitais. CALASS Congresso da Associação Latina para a Análise dos Sistemas de Saúde. Liège, Bruxelas, Bélgica; 2017.

Autor correspondente: Fernando de Rezende Francisco. Escola de Administração de São Paulo, Fundação Getúlio Vargas. Av. Nove de Julho, 2029, $11^{\circ}$ andar, Bela Vista, São Paulo/SP - CEP: 01313-902. Telefone: +55 (11) 3799-7717. E-mail: frfrancisco@gmail.com 


\section{Introdução}

No ano de 2009, houve no país uma chamada pública do Ministério da Saúde (MS) com a Agência Nacional de Vigilância Sanitária (Anvisa) para incentivar a criação de Núcleos de Avaliação de Tecnologias em Saúde (NATS) em hospitais (Assis, 2013). Autores avaliaram o desempenho dos NATS em até três anos após sua implementação (Assis, 2013; Elias et al., 2015; Pereira et al., 2017), mas faltavam dados recentes. Dessa forma, o objetivo do presente estudo foi entender como estavam sendo aplicados os métodos de ATS na tomada de decisão em hospitais sete anos após o advento da chamada pública do MS/Anvisa.

A incorporação de tecnologias em saúde por hospitais afeta o desempenho financeiro e assistencial do sistema de saúde de todo um país, pois esses serviços, sempre entre os mais caros de qualquer sistema, são os principais compradores e usuários dos insumos e processos definidos como tecnologia. Os investimentos nessa área têm um peso importante no desempenho hospitalar, devido ao impacto organizacional a longo prazo sobre as finanças e a qualidade do cuidado. Dessa forma, entender e melhorar os processos de tomada de decisão relativos a investimento nos hospitais é peça-chave para a obtenção de cuidados melhores e mais acessíveis (Wernz et al., 2014).

Sistemas de saúde têm recursos limitados, inviabilizando o atendimento de todas as demandas de uma população. Quando ocorre aumento das necessidades, com o enveIhecimento, maior conhecimento sobre disponibilidade e potencial de novas tecnologias em saúde (Ritrovato et al., 2015), destaca-se a aplicação de ATS como ferramenta para identificar aquelas com maior potencial de benefício para a sociedade (Silva et al., 2010).

A ATS tem aplicação como instrumento de auxílio para a seleção entre alternativas de tecnologias individuais ou conjuntos tecnológicos (duas ou mais tecnologias que devem ser usadas em conjunto para uma finalidade) a serem utilizados para atender a uma mesma necessidade ou problema de saúde (Krauss-Silva, 2004). Seu objetivo é permitir que sistemas ou organizações de saúde (hospitais, clínicas e centros diagnósticos, entre outros) possam aumentar a qualidade do cuidado e o bem-estar do paciente, além de otimizar a relação custo-efetividade, ou seja, a eficiência de produtos para saúde (Nita et al., 2009).

Ainda não está claro em que medida as atividades relacionadas à produção e à difusão dos estudos de ATS têm de fato conseguido influenciar as decisões de incorporação de tecnologias no âmbito da atenção à saúde, no sentido de compatibilizar as evidências disponíveis, as condições de saúde da população e o acesso a tecnologias mais eficientes e socialmente legítimas (Viana et al., 2011). No Brasil, Trindade (2008) destacou que a aplicação de processos de tomada de decisão referentes à incorporação tecnológica nos serviços de saúde do país se torna relevante, pois pode colaborar com o planejamento dos serviços e do sistema.

No âmbito hospitalar, Gagnon et al. (2014) realizaram revisão sistemática da literatura com o objetivo de sintetizar as evidências existentes a respeito dos efeitos e impactos da ATS em hospitais. As buscas iniciais alcançaram pouco mais de 700 artigos. Desses, foram selecionados 18 que atendiam aos critérios aplicados, a saber: estudos qualitativos, quantitativos ou de método misto, com dados empíricos sobre os efeitos ou impactos de ATS realizados em hospital e/ou localmente. Os autores concluíram que a capacidade de avaliar os impactos reais do uso de ATS em hospitais foi limitada, devido, entre outros fatores, à falta de possibilidade de comparar estudos da experiência entre diferentes hospitais (grande heterogeneidade entre os estudos encontrados) e ao fato de que a maioria das avaliações é realizada internamente (a credibilidade seria maior se os estudos fossem realizados por pessoas externas às instituições). Os autores indicam a necessidade de mais estudos para gerar conteúdo suficiente para chegar a uma conclusão sobre a importância do uso de ATS em hospitais.

Outros autores destacam os benefícios financeiros da aplicação de ATS em hospitais. Diversos estudos indicam que ela pode gerar importante economia de recursos financeiros. Em alguns casos, observou-se redução de $50 \%$ de gastos com determinadas tecnologias; em outros, verificou-se economia de recursos na casa de milhões de dólares (Demirdjian, 2015; Lee et al., 2003; Zechmeister \& Schumacher, 2012; Schumacher \& Zechmeister, 2013).

A implementação plena de um núcleo de ATS em um hospital pode enfrentar grandes dificuldades, conforme verificado em países extremamente heterogêneos. Estudo iraniano realizado para identificar barreiras enfrentadas para o estabelecimento dessa ferramenta no país concluiu que a pressão política, a necessidade de respostas rápidas e a falta de uma força de trabalho especializada em ATS foram as ameaças detectadas (Mohtasham et al., 2017). Na Finlândia, estudo de caso sobre a colaboração entre hospitais e a agência nacional finlandesa de ATS analisou 48 relatórios de ATS realizados durante sete anos de colaboração entre as partes. Nesse estudo não foram identificados indicadores claros que demonstrassem o aumento no uso de ATS no processo de tomada de decisão nos hospitais. Apesar da boa qualidade dos relatórios de ATS, sua aplicação foi limitada (Halmesmäki et al., 2016).

Elias \& Araújo (2014) realizaram estudo para entender de que forma as avaliações econômicas em saúde contribuíram para a tomada de decisão na saúde pública do Brasil. Os autores afirmam que a influência dos estudos econômicos na tomada de decisões no nível do estabelecimento municipal da infraestrutura de saúde local foi bastante limitada; por outro lado, ressaltam que o uso da análise de impacto orçamentário desempenhou papel importante no sistema público de saúde. 


\section{Boas práticas em ATS}

Na Europa, o uso da ATS em hospitais já está em fase mais madura de uso, e há maior difusão de seus conceitos e de sua importância. Tal estágio de evolução permitiu que houvesse experiência e massa crítica suficientes para o desenvolvimento de amplo estudo sobre boas práticas de ATS em hospitais. Sampietro-Colom et al. (2016) realizaram estudo para a definição de princípios de boas práticas de ATS em hospitais. O estudo foi conduzido por especialistas europeus que realizaram um amplo trabalho envolvendo revisão de literatura, pesquisas em larga escala, grupo focal, pesquisa Delphi e mais de cem entrevistas presenciais. Ao todo, participaram da definição dos princípios 385 pessoas de 20 diferentes países. De acordo com os autores, os resultados do trabalho da unidade de ATS em hospitais devem ser medidos pelo impacto interno e externo, a curto e médio prazos, bem como o impacto a longo prazo no desempenho do hospital e na saúde das comunidades. Foram definidos 15 princípios orientadores para boas práticas de ATS em hospitais, os quais estão organizados em quatro diferentes dimensões: Processo de Avaliação; Liderança, Estratégia e Parcerias; Recursos; e Impacto (Sampietro-Colom et al., 2016).

\section{Os resultados de uma política no Brasil}

De acordo com Assis (2013), um dos principais objetivos da chamada pública de 2009 foi introduzir a cultura de ATS nos hospitais, por meio da utilização das evidências disponíveis para auxiliar o gestor a tomar decisões quanto à inclusão de novas tecnologias, à avaliação de tecnologias difundidas e ao seu uso racional. Portanto, a chamada pública foi uma forma de indução de política pública para a disseminação da ATS.

No presente trabalho, seguindo os objetivos de outros autores (Assis, 2013; Elias et al., 2015; Pereira et al., 2017), procurou-se estudar o impacto da chamada pública na aplicação da ATS em hospitais no país ao tomar como amostras NATS ligados à Rebrats (Rede Brasileira de Avaliação de Tecnologias de Saúde).

Assis (2013) realizou pesquisa para avaliar o papel específico da ATS na retirada de dispositivos médicos obsoletos que estavam à disposição dos profissionais no Sistema Único de Saúde (SUS), chamada de desincorporação ou exnovation. No trabalho, foi aplicado inquérito aos 24 NATS ligados à Rebrats, existentes no Brasil na época da coleta de dados, setembro de 2012, dos quais 11 responderam. Constatou-se que o desinvestimento era exercido por poucos centros e, mesmo assim, de forma incipiente. $O$ estudo permitiu verificar que a ATS não vinha sendo usada como ferramenta de análise de tecnologias já disponíveis para uso nos hospitais.

Elias et al. (2015) realizaram estudo para identificar os desafios para a utilização de ATS em hospitais de ensino. No estudo, aplicaram um questionário (a distância) para autopreenchimento, utilizando-se o programa FormSUS, gerido pelo Departamento de Informática do SUS (Datasus), a gesto- res dos 24 hospitais que receberam incentivo público para a implantação de NATS, de acordo com a chamada pública realizada pelo MS/Anvisa em 2009. O questionário ficou disponível para preenchimento no período de 29 de agosto a 14 de outubro de 2011. Dos 24 NATS convidados, 20 responderam ao questionário. Os resultados indicaram que nos hospitais de ensino houve, até aquele momento, pouca influência dos NATS em processos assistenciais e tomadas de decisão, indicando sua subutilização.

Pereira et al. (2017) entrevistaram presencialmente gestores de 23 NATS (todos criados e financiados pela chamada pública do MS/Anvisa de 2009), para descrever a situação e experiência dos primeiros NATS do Brasil em seu período inicial de existência. As autoras planejavam entrevistar os 24 gestores de NATS criados pela chamada pública, no entanto um deles se recusou a colaborar com o estudo. A coleta de dados foi realizada nos anos de 2011 e 2012, portanto três anos após a implementação dos NATS. As autoras relataram disparidades regionais de carga de trabalho, produção e treinamento técnico entre os núcleos. E concluíram que a criação dos NATS foi uma iniciativa positiva, pois aumentou a capacidade dos profissionais envolvidos e levou à criação de novos NATS no país. No entanto, indicaram que faltam investimentos contínuos que permitam aos profissionais receberem educação continuada e desenvolverem atividades relevantes de ATS.

Sendo assim, para o presente estudo, foi definida a seguinte pergunta de pesquisa: "como são aplicados os métodos de ATS na tomada de decisão em hospitais?". Buscou-se atualizar dados sobre a situação dos NATS vinculados à Rebrats contextualizando-os com aspectos importantes dos conceitos abordados nos princípios de boas práticas de ATS em hospitais de Sampietro-Colom et al. (2016), com adaptações à realidade do Brasil.

\section{Métodos}

\section{Amostra}

Para a definição da amostra, partiu-se do grupo formado pelos NATS de hospitais vinculados à Rebrats. No momento do estudo, 27 núcleos atendiam a essa condição. Como critério de seleção, foram aplicadas duas variáveis: (i) localização a partir da região geográfica do país e (ii) produtividade do NATS de acordo com os trabalhos divulgados no Sisrebrats (Sistema de Informação da Rede Brasileira de Avaliação de Tecnologia em Saúde). Os núcleos foram separados por região geográfica em que estavam localizados, e para cada região foram selecionados dois NATS: aquele com maior quantidade de trabalhos publicados no Sisrebrats e aquele com menor quantidade; nas regiões em que essa regra não fosse suficiente para diferenciá-los, admitiu-se NATS com igual produtividade. Ao todo, participaram do estudo 11 NATS. Para a região Sudeste, que compreendia cerca de 
40\% dos NATS de todo o grupo, foram selecionados três núcleos, com a intenção de buscar maior representatividade da seleção. No estudo foi realizada, portanto, amostragem não probabilística por conveniência, estratificada por região geográfica do país e por produtividade identificada.

\section{Método}

Os gestores dos NATS selecionados foram entrevistados e os áudios das entrevistas foram gravados e transcritos para posterior análise. As entrevistas foram presenciais, por videoconferência ou por telefone, de acordo com a disponibilidade do entrevistado.

\section{Instrumento de coleta}

As entrevistas foram baseadas em roteiro semiestruturado preparado utilizando os 15 princípios orientadores para boas práticas de ATS em hospitais, descritos por Sampietro-Colom et al. (2016). Após a adaptação dos 15 princípios, o instrumento de coleta foi definido com 32 itens divididos em seis categorias: (i) Histórico do NATS; (ii) O processo de Avaliação de Tecnologias em Saúde; (iii) Liderança, estratégia e parcerias; (iv) Recursos; (v) Impacto; (vi) Planejamento.

\section{Instrumento de análise de dados}

O processo de codificação e categorização foi realizado para todas as entrevistas. Durante o processo de análise dos resultados, as codificações foram analisadas e reanalisadas, e o mesmo foi feito com o conteúdo das entrevistas, que foram revisitadas diversas vezes. Esse processo de comparação constante e codificação faz parte da metodologia adotada (Bandeira-de-Mello \& Cunha, 2003). Notas de análise relacionadas à criação de códigos, categorias e propriedades foram elaboradas durante o processo com o uso do software ATLAS.ti (Bandeira-de-Mello, 2006), para ajudar na aplicação padronizada dos códigos e na organização das abstrações necessárias no processo.

\section{Resultados}

Foram identificadas diferenças importantes na composição dos NATS entrevistados, desde núcleos que se resumem a um único profissional com dedicação de horas fora de seu expediente de atividade assistencial, até outros muito bem estruturados e altamente ligados ao processo de tomada de decisão institucional. No entanto, em todos há oportunidades de melhoria e aspectos a serem mais bem desenvolvidos.

A seguir, são apresentados os temas mais mencionados pelos gestores durante as entrevistas, suas descrições e impactos nas atividades dos NATS.

\section{Origem dos NATS e seu trabalho em rede}

A chamada pública do MS/Anvisa no ano de 2009 (Assis, 2013) foi fundamental para a criação de muitos dos NATS participantes da pesquisa. Anteriormente à chamada, houve a definição de políticas de criação da Rede de Hospitais Sentinela e de suas áreas de Gerenciamento de Risco. Diversos entrevistados mencionaram que os profissionais envolvidos nessas duas áreas foram também envolvidos na criação dos NATS nos hospitais e já traziam consigo a capacidade de trabalho em rede e no estabelecimento de novos processos e rotinas nos hospitais. No entanto, a experiência prévia com o trabalho em rede não foi suficiente para o estabelecimento pleno do trabalho em rede com os NATS.

\section{Incorporação e desincorporação}

Os gestores entrevistados demonstraram ter conhecimento sobre as possibilidades de aplicação das análises de ATS para a incorporação e, também, desincorporação de tecnologias nos hospitais em que trabalham e nos sistemas de saúde nos quais estão inseridos (em âmbito municipal, estadual e federal). Entretanto, não se constatou na prática que os NATS vinham desempenhando análises dessa natureza para tecnologias dos hospitais nos quais estão inseridos, e alguns deles atuavam em projetos para secretarias ou para o Ministério da Saúde.

\section{Financiamento}

Não há financiamento direcionado ao NATS e os núcleos não têm orçamento próprio, o que dificulta o planejamento de suas ações, desenvolvimento e retenção de profissionais e seu bom funcionamento. As verbas são oriundas de editais e cartas-acordo, e muitos dos NATS ainda existem por esforço pessoal de profissionais abnegados que enxergam a importância deles e acreditam que em algum momento passarão a ter recursos para a difusão das análises de ATS em seus estabelecimentos e nos sistemas de saúde. Dessa forma, mesmo após a indução de políticas públicas e o financiamento inicial promovido por chamada pública, não houve de fato a institucionalização do NATS nos hospitais.

\section{Análises econômicas}

Os NATS praticamente não realizam análises econômicas, simples ou elaboradas, e pouco utilizam dados do hospital para entendimento do impacto orçamentário da incorporação de tecnologias. Como métodos sistemáticos de trabalho no NATS, foram mencionados pelos gestores entrevistados mini-ATS, parecer técnico-científico, revisões sistemáticas e saúde/medicina baseada em evidências. Para grande parte dos coordenadores de NATS entrevistados, as principais atividades dos núcleos são análises de literatura científica para entendimento de segurança e eficácia relacionada a determinada tecnologia.

Cabe aqui ressaltar que praticamente não há organização dos dados de atendimento dos hospitais e não se conhece o perfil epidemiológico do público atendido no estabeleci- 
mento, o que poderia ser a base norteadora para o estabelecimento de uma agenda de avaliações pelo NATS.

\section{Pessoal}

Faltam profissionais com formação em economia da saúde nos hospitais. Essa circunstância foi apontada como um dos principais desafios enfrentados pelos NATS. Mesmo não atuando diretamente no núcleo, os profissionais com conhecimentos na área poderiam aplicar conceitos de economia da saúde para subsidiar as mais diversas tomadas de decisão às quais são submetidos diariamente. A afinidade com os conceitos de ATS dos envolvidos com os NATS surgiu como fator determinante para a aproximação do profissional com o núcleo, bem como para a sua permanência. Alguns profissionais percebem a importância do tema à medida que surgem situações em suas áreas de trabalho para as quais necessitam de análises econômicas para amparar a tomada de decisão. Ter equipe de profissionais com dedicação exclusiva é fator apontado como preponderante para o sucesso nos trabalhos dos NATS. Os colaboradores dos NATS comumente têm sua ocupação principal no hospital nas áreas assistencial, de pesquisa ou administrativa, e suas atividades no NATS são secundárias: dos 11 entrevistados, três têm equipe mista dividida entre pessoal totalmente dedicado e parcialmente dedicado, e os oito restantes têm equipe parcialmente dedicada. Portanto, nenhum NATS tem toda sua equipe trabalhando com dedicação exclusiva ao núcleo.

\section{Modus operandi}

Os NATS trabalham com modelos de relatórios e pareceres, mas dificilmente têm procedimentos operacionais padrão que definam o papel de cada membro, processos, fluxo de trabalho, entre outros, mas há exceções de núcleos com esses aspectos mais desenvolvidos. A equipe tênue, sem dedicação exclusiva, e as incertezas para contratação e retenção de pessoas talvez expliquem a aparente falta de mão de obra para redigir tais procedimentos, ou mesmo o motivo pelo qual não são priorizados quando há mão de obra disponível. Não há padrão de processos e funcionamento dos NATS, nem mesmo sobre sua localização na hierarquia dos hospitais.

\section{Apoio}

A falta de apoio e orientação da direção do hospital pode ser o fator responsável por alguns NATS ainda não estarem formalizados ou mesmo ainda não estarem efetivamente ativos em seus hospitais. Por outro lado, há exemplos de apoio e incentivo da direção de hospitais aos NATS indicando forte relação desse fator com a maior atividade do núcleo.

\section{Cultura}

Relatou-se bastante durante as entrevistas a falta de cultura de ATS nas instituições, ou melhor, dos profissionais que atuam nas instituições.
A dita "cultura em ATS" nos hospitais pode ser definida como a utilização de conceitos de ATS e economia da saúde para embasar ações e subsidiar a tomada de decisão nas mais diversas áreas do estabelecimento, não necessariamente apenas naquelas situações específicas de elaboração de estudos econômicos. Também pode ser entendida como o conhecimento pela maioria dos profissionais da instituição dos conceitos de ATS.

Pode haver relação entre a falta de cultura de ATS nos hospitais e a falta de profissionais capacitados: o desconhecimento sobre o assunto faz com que ele seja ignorado, ou mesmo tenha pouca importância para alguns profissionais.

\section{Planejamento}

Há muito pouco planejamento das atividades dos núcleos. Isso não está vinculado à falta de preparo dos envolvidos, mas a outros fatores tais como falta de orçamento próprio e de pessoas dedicadas, de sua definição como instância obrigatória na análise de incorporação ou desincorporação de tecnologias e ausência de procedimentos operacionais padrão.

\section{Discussão}

O processo de criação dos NATS surgiu com a indução governamental a partir da qual se esperava a criação das áreas nos serviços, desenvolvimento da cultura de ATS nos hospitais, formação de pessoal qualificado, pleno apoio para estudos de ATS para suprir demandas internas e externas à instituição, enfim, um processo interligado que criaria um ciclo de ações que se retroalimentariam para a aplicação da ATS no setor. Na prática, o que se viu foi o estímulo financeiro pontual que não foi suficiente para fazer com que o ciclo pudesse se manter.

A falta da cultura de ATS, a qual foi estimulada pela chamada pública de 2009 (MS/Anvisa), foi apontada pelos NATS como um dos desafios a serem superados para a evolução do tema nos hospitais. Cicchetti et al. (2008) sugeriram que a implementação de uma unidade interna de ATS em hospital pode ser útil para a difusão da cultura de ATS e da medicina baseada em evidências e para criar um contexto organizacional positivo para o uso dessas ferramentas no apoio à prática clínica e à tomada de decisão gerencial. A grande necessidade de formação de pessoas em ATS informada por praticamente todos os NATS entrevistados também aponta para a falta de cultura de ATS, pois os profissionais da saúde dessas instituições, de maneira geral, desconhecem o tema, mesmo após anos de esforços empreendidos para sua disseminação. Sendo assim, a criação de uma unidade interna de ATS, o NATS, não constitui ação suficiente para a difusão da cultura de ATS, nem para a real inclusão do núcleo no processo de tomada de decisão clínico e gerencial.

Quando o foco de análise está no conhecimento dos profissionais diretamente ligados aos NATS, verificou-se amplo 
conhecimento sobre ATS e sua importância para amparar as decisões de incorporação e desincorporação. A não aplicação dos processos de ATS, portanto, não parece estar vinculada à falta de conhecimento dos profissionais diretamente ligados aos NATS.

Faltam indicadores sobre o trabalho dos NATS e não há coleta estruturada de dados sobre o desempenho do pessoal envolvido, nem sobre os resultados das atividades dos núcleos - por conseguinte, não há planilhamento, acompanhamento e análise. A falta de dados dificulta o desenvolvimento do NATS, pois não se justifica a alocação de recursos de qualquer natureza na área. Tal situação leva a um paradoxo: sem recursos, o NATS não consegue apresentar resultados; sem resultados, o NATS não consegue mais recursos. E mais, sem recursos, o NATS não consegue desenvolver trabalhos que demonstrem às demais áreas do hospital quão importante é o papel que ele pode desempenhar na instituição.

Os dados obtidos durante as entrevistas indicam que há fragilidade na influência dos NATS para a tomada de decisão em hospitais. Conforme mencionado anteriormente, a maioria das instituições consultadas não realiza avaliação econômica completa, atendo-se apenas a estudos mais simples, tais como descrição de tecnologia e recomendação técnico-científica. Novaes \& Soárez (2016) apontam que características similares puderam ser encontradas na Citec (Comissão para Incorporação de Tecnologias do Ministério da Saúde), de 2008 a 2010, e na Conitec, de 2012 a 2015. Respectivamente nos períodos mencionados, somente $11,6 \%$ dos 103 estudos realizados pela Citec foram análises econômicas, e na Conitec somente $11,1 \%$ das tecnologias recomendadas para incorporação apresentaram avaliação econômica completa com o cálculo da razão de custo-efetividade incremental. Portanto, a realidade das atividades dos NATS atualmente é similar àquela descrita a partir de 2008; em cerca de 10 anos pouca coisa mudou nas características das avaliações que são elaboradas.

O desenvolvimento de indicadores de desempenho para as atividades e pessoal dos NATS poderia ser um dos pontos de apoio da Rebrats, ou mesmo da Empresa Brasileira de Serviços Hospitalares (Ebserh), aos núcleos. Um conjunto de indicadores serviria como ferramenta de orientação para a definição de atividades, organização e planejamento pelos NATS e fortaleceria o trabalho em rede, estimulando e tornando mais acessível a troca de experiências entre os núcleos.

Dados estruturados sobre os atendimentos do estabelecimento, somados a pessoal capacitado no NATS, criariam base para a adequada contextualização de estudos de ATS para cada estabelecimento, mitigando variações no contexto econômico e social inter-hospitais no Brasil. Assim, corrobora-se o que foi apontado por Cicchetti et al. (2008): o valor do uso de determinada tecnologia médica varia conforme recursos e competências disponíveis em cada organização de saúde, e seu valor deve ser julgado de acordo com contextos organizacionais específicos, sem detrimento da importância de ATS como suporte para a tomada de decisão política em nível macro; se a decisão deve ser tomada em nível hospitalar, as evidências e dados devem ser coletados e analisados no contexto da organização.

A partir das informações coletadas nas entrevistas, as iniciativas da Rebrats e da Ebserh dão apoio aos NATS com treinamentos e capacitação de pessoal - e são iniciativas valorizadas pelos integrantes dos núcleos. No entanto, as análises de outras variáveis já descritas aqui mostram que nem a Rebrats nem a Ebserh conseguiram efetivar a atuação em rede entre os núcleos. Há espaço para o fortalecimento do intercâmbio de conhecimento desde a base estrutural dos núcleos até a disseminação de resultados de análises econômicas e seu uso para amparar as tomadas de decisão nas instituições.

A concreta estruturação da ATS como etapa fixa no processo de tomada de decisão em hospitais parece ser o resultado de diversas iniciativas organizadas. Não basta investir recursos financeiros; tampouco são suficientes ações pontuais de capacitação. A contribuição deste estudo baseia-se na definição de macroetapas interligadas, determinando-se um fluxo, de tal maneira que o resultado de uma delas seja subsídio para o início de outra (ou de outras), a fim de gerar um círculo virtuoso de aplicação da ATS em hospitais. As macroetapas definem o nível estratégico e devem ser transformadas localmente em ações táticas e operacionais para que possam se tornar atividades concretas em cada NATS. A Figura 1 ilustra essa contribuição.

O fluxo de ações idealizadas, conforme a Figura 1, sugere que o ciclo deve funcionar da seguinte maneira: a inclusão efetiva do NATS como uma das instâncias obrigatórias do processo de tomada de decisão sobre incorporação ou desincorporação de tecnologias nos hospitais depende de núcleos formados por pessoal capacitado e dedicado ao NATS. A realização de estudos de ATS no hospital habilita o NATS a trabalhar em rede, na qual se pressupõe a troca de experiências e estudos. $\mathrm{O}$ resultado de todo esse processo deve ser mensurado para permitir avaliar o desempenho das atividades e tomar medidas corretivas, ou mesmo justificar os investimentos na unidade. A partir de investimentos, o núcleo terá condições plenas de estrutura, recursos materiais e de pessoal para cumprir seu papel. Um dos papéis esperados do NATS é a capacitação dos profissionais do hospital, quer sejam da assistência ou da gestão, para que entendam de que forma a ATS pode ser aplicada, seus benefícios do ponto de vista financeiro, clínico e de qualidade, e até mesmo como isso gerará impacto para os pacientes e para a sociedade. Assim, com a capacitação, haverá disseminação da cultura de ATS, que pode ser entendida como a utilização de evidências baseadas em conceitos de ATS e em estudos de economia da saúde para auxiliar gestores, clínicos e outros profissionais do 


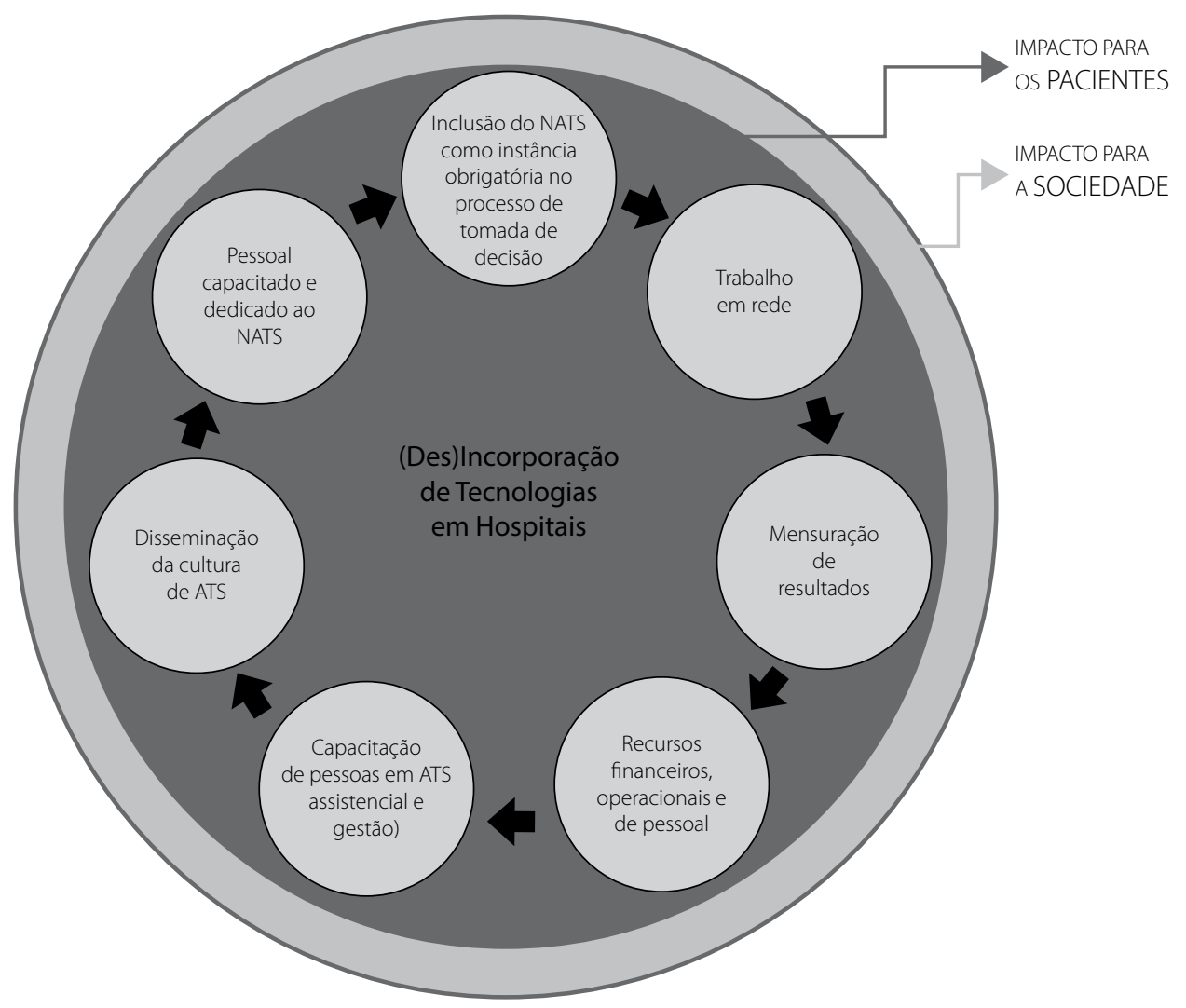

Figura 1. Esquema das macroetapas necessárias ao pleno funcionamento do NATS em hospitais.

hospital a tomarem decisões quanto à inclusão de novas tecnologias, à avaliação de tecnologias difundidas e ao seu uso racional - o que volta a remeter ao tema do valor da ATS para o paciente e para a sociedade. E, por fim, fechando o último elo do ciclo, a capacitação de profissionais e a disseminação da cultura de ATS farão com que aqueles que se identifiquem com o tema sejam convidados a integrar o NATS ou espontaneamente o procurem, criando um círculo virtuoso no processo de ATS nos hospitais.

\section{Conclusões}

A partir do material coletado nas entrevistas e analisado para este estudo, pode-se concluir que a ATS não é aplicada no processo de tomada de decisão em hospitais, com recursos de pessoal e financeiros insuficientes e processos operacionais estabelecidos fracamente ou não estabelecidos.

Os NATS podem auxiliar tanto em questões relacionadas à prática clínica quanto em tomadas de decisão gerenciais. No entanto, parece não haver alinhamento entre os objetivos dos núcleos e o apoio que recebem em nível institucional, ou mesmo eventuais apoios externos (governamental ou não governamental). Tampouco há padrão bem estabele- cido para a aplicação da ATS: o tipo de trabalho executado atualmente pelos núcleos é bastante variável.

Do ponto de vista organizacional, não há indicadores de desempenho padronizados, e muitos núcleos sequer têm ferramentas para medir seus resultados (quais e quantos estudos realizam e quais seus impactos para o hospital ou sistema de saúde - quando o estudo é realizado para secretarias de saúde ou SUS). Soma-se a isso a pouca cultura em ATS nas instituições, o que dificulta o entendimento de que o investimento no NATS pode amplificar os recursos disponíveis para as áreas assistenciais, ampliando o acesso aos serviços e melhorando a equidade. Os recursos financeiros aplicados no NATS deveriam ser entendidos como investimentos, não como custeio, pois deveriam gerar valor à cadeia da saúde.

O sucesso dos NATS para a incorporação e desincorporação de tecnologias em saúde em hospitais depende de uma cadeia de ações estratégicas, conforme apresentado na Figura 1, e não apenas de estímulos pontuais.

A sugestão deste estudo é que seja criado um círculo virtuoso propício para o uso de ATS para subsidiar a tomada de decisão. A falta de qualquer uma das macroetapas estratégicas apresentadas enfraquece, ou mesmo inviabiliza, a plena aplicação da ATS para a tomada de decisões em hospitais. 


\section{Agradecimentos}

Os autores agradecem aos gestores das 11 instituições participantes, que entenderam a importância desta pesquisa e dedicaram seu tempo para contribuir com este trabalho.

\section{Referências bibliográficas}

Assis EC. O papel da avaliação de tecnologias em saúde (ATS) na retirada de dispositivos médicos obsoletos no Sistema Único de Saúde (SUS) [dissertação]. Rio de Janeiro: Escola Nacional de Saúde Pública Sergio Arouca; 2013

Bandeira-de-Mello R. Software em pesquisa qualitativa. In: Godoy CK, Bandeira-de-Mello R, Silva AB, orgs. Pesquisa Qualitativa em Estudos Organizacionais: Paradigma, Estratégias e Métodos. São Paulo: Saraiva; 2006. p. 429-60.

Bandeira-de-Mello R, Cunha CCA. Operacionalizando o método da Grounded theory nas pesquisas em estratégia: técnicas e procedimentos de análise com apoio do software ATLAS/TI. In: Encontro de Estudos em Estratégia (3ES), 1., 2003, Curitiba. Anais... Curitiba-PR: ANPAD; 2003. v. 1, p. 1-18.

Cicchetti A, Marchetti M, Dibidino R, Corio M; on behalf of HTAi's Hospital Based Sub Interest Group: Hospital based HTA. Hospital based health technology assessment. World-Wide Survey. 2008. Available at: http:// www.htai.org/fileadmin/HTAi_Files/ISG/HospitalBasedHTA/2008Files/ HospitalBasedHTAISGSurveyReport.pdf. Accessed on: 06/01/2016.

Demirdjian G. A 10-year hospital-based health technology assessment program in a public hospital in Argentina. Int J Technol Assess Health Care. 2015;31(1/2):103-10.

Elias FTS, Leão LS, Assis EC. Avaliação de tecnologias em hospitais de ensino: desafios atuais. Tempus Actas de Saúde Coletiva. 2015;9(3):147-58.

Elias FT, Araújo DV. How health economic evaluation (HEE) contributes to decision-making in public health care: the case of Brazil. Z Evid Fortbild Qual Gesundhwes. 2014;108(7):405-12.

Gagnon MP, Desmartis M, Poder T, Witteman W. Effects and repercussions of local/hospital-based health technology assessment (HTA): a systematic review. Syst Rev. 2014;3:129.

Halmesmäki E, Pasternack I, Roine R. Hospital-based health technology assessment (HTA) in Finland: a case study on collaboration between hospitals and the national HTA unit. Health Res Policy Syst. 2016;14:25.

Krauss-Silva L. Avaliação tecnológica em saúde: questões metodológicas e operacionais. Cad Saúde Pública. 2004;20(Suppl 2):S199-S207.
Lee RC, Marshall D, Waddell C, Hailey D, Juzwishin D. Health technology assessment, research, and implementation within a health region in Alberta, Canada. Int J Technol Assess Health Care. 2003;19(3):513-20.

Mohtasham F, Majdzadeh R, Jamshidi E. Hospital-based health technology assessment in Iran. Int J Technol Assess Health Care. 2017;33(4):529-33.

Nita ME, Secoli SS, Nobre M, Ono-Nita SK. Métodos de pesquisa em avaliação de tecnologias em saúde. Arq Gastroenterol. 2009;46(4):252-5.

Novaes HMD, Soárez PC. Organizações de avaliação de tecnologias em saúde (ATS): dimensões do arcabouço institucional e político. Cad Saúde Pública. 2016;32(Suppl 2):S1-S14.

Pereira CCA, Rabello RS, Elias FTS. Hospital-based health technology assessment in Brazil: an overview of the initial experiences. Intl J Technol Assess Health Care. 2017;33(2):227-31.

Ritrovato M, Faggiano FC, Tedesco G, Derrico P. Decision-oriented health technology assessment: one step forward in supporting the decisionmaking process in hospitals. Value Health. 2015;18(4):505-11.

Sampietro-Colom L, Lach K, Pasternack I, Wasserfallen JB, Cicchetti A, Marchetti M, et al. Guiding principles for good practices in hospitalbased health technology assessment units. Int J Technol Assess Health Care. 2016;31(6):1-9.

Schumacher I, Zechmeister I. Assessing the impact of health technology assessment on the Austrian healthcare system. Int J Technol Assess Health Care. 2013;29(1):84-91.

Silva EN, Silva MT, Elias FTS. Sistemas de Saúde e Avaliação de Tecnologias em Saúde. In: Nita ME, Campino ACC, Secoli SR, Sarti FM, Nobre M, Costa AM, et al., eds. Avaliação de tecnologias em saúde. Porto Alegre: Artmed; 2010. p. 419-32.

Trindade E. A incorporação de novas tecnologias nos serviços de saúde: o desafio da análise dos fatores em jogo. Cad Saúde Pública. 2008;24(5):951-64.

Viana AL, Nunes AA, Silva HP. Complexo produtivo da saúde, desenvolvimento e incorporação de tecnologias. In: Ibañez N, Elias PEM, Seixas PHD, orgs. Política e gestão pública em saúde. São Paulo: Hucitec/Cealag; 2011. p. 75-101.

Wernz C, Zhang H, Phusavat K. International study of technology investment decisions at hospitals. Ind Manag Data Syst. 2014;114:568-82.

Zechmeister I, Schumacher I. The impact of health technology assessment reports on decision making in Austria. Int J Technol Assess Health Care. 2012;28(1):77-84. 